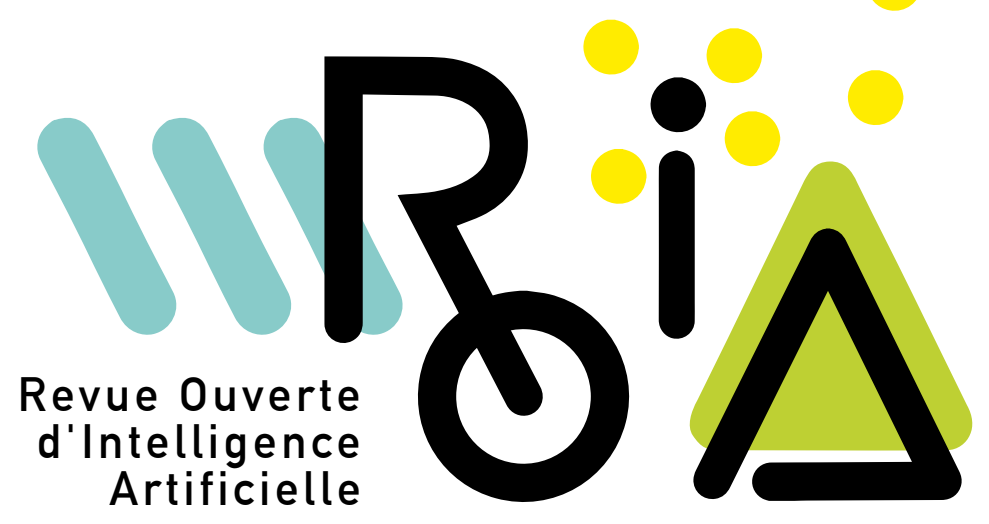

Christophe Roche, Maria Papadopoulou

Rencontre entre une philologue et un terminologue au pays des ontologies

Volume 1, no 1 (2020), p. 43-70.

<http://roia.centre-mersenne.org/item?id=ROIA_2020_1_1_43_0>

(C) Association pour la diffusion de la recherche francophone en intelligence artificielle et les auteurs, 2020, certains droits réservés.

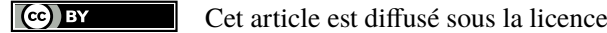

Creative Commons Attribution 4.0 International License.

http://creativecommons.org/licenses/by/4.0/ 


\title{
Rencontre entre une philologue et un terminologue au pays des ontologies
}

\author{
Christophe Roche $^{a}$, Maria Papadopoulou ${ }^{b}$ \\ ${ }^{a}$ Équipe Condillac, Laboratoire Listic, Université Savoie Mont-Blanc Campus \\ Scientifique, 73376 Le Bourget du Lac cedex, France. \\ E-mail : christophe.roche@univ-savoie.fr \\ ${ }^{b}$ Knowledge Engineering \& Terminology Research Centre, Liaocheng University, No. 1 \\ Hunan Road, Liaocheng City Liaocheng, Shandong 252059, P. R. China. \\ E-mail: maria.papadopoulou@ketrc.com.
}

\begin{abstract}
La classification des objets, le choix des termes et leur définition au regard des connaissances du domaine, sont des préoccupations constantes en Humanités Numériques (HN). Dans ce cadre, et dans le contexte du partage et de l'interopérabilité des données sur le Web, les ontologies au sens de l'Ingénierie des Connaissances (IC) constituent une des avancées les plus intéressantes. Mais les principes sous-jacents à la conceptualisation d'un domaine tels que l'entendent l'IC et les HN ne sont pas nécessairement les mêmes. À cela s'ajoute un problème de Terminologie en tant que discipline souvent sous-estimée aussi bien par les uns que par les autres. Cet article s'intéresse à la façon de procéder des experts dans leur "définition des choses" et dans la manière de les nommer. Cela nous a conduits à définir une méthode outillée de construction de terminologies dont le système conceptuel est une ontologie formelle, dans le respect des normes ISO et des standards du W3C. Cette méthode est illustrée à l'aide du premier dictionnaire ontoterminologique des vêtements de la Grèce antique.

Mots-CLÉs. - Représentation des connaissances, ontologie, terminologie, ontoterminologie, méthodologie, humanités numériques, lexicographie historique, ontologie des vêtements de la Grèce antique, dictionnaires électroniques, normes ISO, standards W3C, éditeur d'ontologies et de terminologies, TEDI. .
\end{abstract}

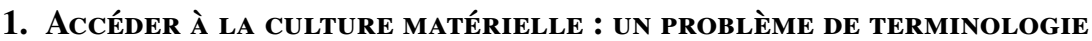

Aborder la culture matérielle $\mathrm{e}^{(1)}$ du monde antique sous un angle purement terminologique est une approche relativement nouvelle. On a généralement recours à des dictionnaires historiques pour la recherche de la signification de termes dénotant des objets matériels des cultures passées. Un des premiers dictionnaires historiques de référence, le dictionnaire Grec-Anglais Liddell, Scott et Jones (largement connu des

(1) i.e. les objets de la vie courante utilisés par un groupe humain à une période et dans un lieu donnés qui le distinguent des autres groupes : arts, bâtiments, armes, ustensiles, machines, coiffures, vêtements, bijoux, etc. 
étudiants et chercheurs en études classiques sous le nom de LSJ) publié la première fois en $1848^{(2)}$, suit ce qui a été décrit comme l' « approche historique » pour laquelle chaque mot (entrée du dictionnaire) est décrit par l'histoire de ses emplois ${ }^{(3)}$, comprenant des équivalents de traduction, sans inclure de définitions (onto)logiques. Les textes tiennent également une place importante, et il semble naturel d'utiliser les termes en usage dans la communauté. Cependant, cette approche n'a, en dehors des problèmes évidents de diachronie, qu'un succès limité dans les domaines des vêtements et de la poterie de la Grèce antique, n'étant pas certain de ce que les termes peuvent dénoter : «Although literary sources and temple inventories have preserved the names of many garments, few can be identified with certainty in exact representations » [84], ce que corroboraient déjà en leur temps Richter et Milne, conservateurs au Musée Metropolitan de New York [71], mais aussi l'historien des vêtements de la Grèce antique K. Stears [87], et M. Lee [53] auteur d'une récente monographie sur les vêtements grecs anciens : «The conventional typology and terminology are employed here purely for convenience ».

Le résultat est un «babélisme terminologique » [7] qui nuit au partage des connaissances et à la communication entre experts comme le souligne Delaporte [25], anthropologue travaillant sur la classification de la culture matérielle : «Le flou terminologique qui règne dans les études consacrées au costume, et qui est peut-être une des causes des difficultés de communication entre chercheurs ». Les experts sont alors nombreux à réclamer une « unité terminologique » transcendant les barrières linguistiques et culturelles : «il manque une certaine unité dans le vocabulaire employé » [18] et tentent, dans leurs travaux à « [...] répondre au besoin d'un système de dénomination transculturelle des pièces vestimentaires. » [7]. Ce sont autant d'arguments qui militent pour une approche normative des terminologies comme le montre, pour un domaine différent, la discussion « On the standardization of Ceramic Terminology » publiée en 1970 dans la revue Current Anthropology [49]. Won-Yong Kim (1970), archéologue spécialisé dans l'archéologie asiatique, résume bien la problématique : «As Claerhout points out, standardization of the terminology for the shapes of ceramic vessels might be helpful. [...] I feel, nevertheless, that it would help if we could work out a list of standard vessel shapes, clearly defined and illustrated, and a set of terms for them that is perhaps entirely different from, and so cannot be confused with, the terms in common popular use ». Un besoin de normalisation qui ne se limite pas aux catégories vestimentaires, ni aux seules cultures européennes. Kirkland Lothrop [50], archéologue et anthropologue spécialisé dans les études centraméricaines et sud-américaines, déclare dans son ouvrage sur la poterie du Costa Rica et du Nicaragua : «An intelligent discussion of pottery shapes is rendered more difficult by lack of definitive nomenclature ».

\footnotetext{
${ }^{(2)}$ Les dictionnaires standards du Grec ancien sont bilingues et leurs premières éditions datent $\mathrm{du} \mathrm{XIX}^{\mathrm{e}}$ siècle. Ils ont été continuellement réimprimés et plus récemment mis en ligne, consultables sur différents sites Web : Grec-Français (Bailly [6]; Chantraine [19] se concentrant sur l'histoire des mots); Grec-Anglais [55], Anglais-Grec [94], Grec-Allemand [65]. Citons également Eulexis (https: //outils.biblissima.fr/en/eulexis/index.php), lemmatiseur du Grec ancien, qui a été conçu pour pouvoir consulter les trois dictionnaires les plus utilisés pour la traduction du Grec ancien en Anglais (LSJ), Français (Bailly-abrégé), Allemand (Pape).

(3) [35].
} 
Dans le domaine de la culture matérielle, les objets sont les témoins du passé et nous avons besoin de les nommer pour en parler. Les noms sont donnés aux choses par commodité, exprimant parfois une propriété, une caractéristique, une fonction de l'objet. Ils peuvent être métaphoriques, motivés par une similitude ou une analogie conceptuelle, ou empruntés au grec ou au latin leur conférant, par le statut de ces langues, une certaine autorité. Si le nom des choses change à travers le temps et l'espace, ils peuvent également donner lieu, en synchronie, à des interprétations différentes selon leurs contextes discursifs. Se reposer sur l'étude des seuls textes n'est non seulement pas suffisant, mais potentiellement source d'erreurs. Notre compréhension des termes doit provenir avant tout de notre connaissance des objets qu'ils désignent [36].

\section{NOMMER LES CHOSES}

\subsection{Contexte : les vêtements de la Grèce AntiQue}

Parmi les objets culturels, les vêtements tiennent une place particulière. Ce n'est pas surprenant, car l'habillement est l'une des expressions fondamentales de l'être humain, tant individuellement que collectivement. En tant que telle, son étude est fondamentale pour comprendre toute culture ou société : «l'histoire du costume entretient des liens très étroits avec l'histoire des sociétés » [37]. En effet, l'habillement est étroitement lié à des aspects essentiels tels que le sexe, l'âge, le rang, la profession, la religion, mais aussi des matériaux, techniques et savoir-faire d'une époque donnée, etc. la liste est longue. Rares sont les disciplines qui ne prennent pas son étude en compte d'une manière ou d'une autre : philosophie [83], sémiotique [10, 38], anthropologie [25], histoire sociale, i.e. celle qui s'intéresse à la signification du vêtement du point de vue sociologique [17], histoire des techniques [8] ${ }^{(4)}$.

Malheureusement, il existe peu de traces de vêtements de la Grèce antique, pour autant qu'il en reste encore des preuves matérielles à ce jour. Les conditions environnementales en Grèce continentale, ainsi que la nature hautement périssable des matériaux utilisés (fibres végétales, animales, peaux, fourrures) entraînent inévitablement la perte de la majeure partie de cet aspect important de la culture Grecque. Néanmoins, les vêtements de la Grèce antique ont une présence «vivante » dans presque tous les textes et les objets datant de cette période qui, conservés dans les collections des musées du monde entier, en portent les représentations : bas-reliefs, sculpture, peintures, peintures sur vases, pièces de monnaie, etc.

\subsection{OBJECTIFS DU PROJET}

Il ne peut y avoir de terminologies des objets culturels sans une connaissance précise des objets que les termes désignent, comme le rappellent Gras [36] et WonYong Kim [49] dans son commentaire à propos de l'article «On the standardization of Ceramic Terminology » : il faut être capable dans un premier temps de clairement

\footnotetext{
(4) Voir aussi [66]. Pour des recherches récentes consacrées à la tenue vestimentaire grecque (y compris les questions de terminologie), voir [67, 58, 34, 51, 52, 53, 57, 56, 74, 75, 20].
} 
définir les connaissances du domaine : «standard vessel shapes, clearly defined and illustrated ». Le problème abordé ici est celui des experts confrontés à nommer les choses et à définir les termes qui les désignent au regard de ce qu'elles sont. Clairement identifier l'objet désigné par un terme donné, par exemple que le vêtement figurant sur tel vase est une chlamyde, nécessite de connaître de façon précise la nature de l'objet représenté. La définition des termes s'exprime alors sous la forme d'une combinaison de caractéristiques, correspondant à un concept, traduisant des propriétés physiques ou fonctionnelles de l'objet : «avec manches », « sans coutures », etc.

La dimension diachronique n'a pas été prise en compte car on s'est intéressé à une époque donnée, correspondant aux périodes Classique et Hellénistique, pour laquelle les «primitives de connaissance » que nous avons prises en compte pour la définition des concepts des vêtements de la Grèce antique peuvent être considérées comme «stables ».

L'objectif n'est pas ici la construction de terminologies à partir de corpus de textes dont l'intérêt, en particulier lorsqu'on ne dispose pas d'experts, n'est plus à démontrer lorsqu'on s'intéresse à l'identification de termes et de leurs différents sens en usage discursif. Il ne s'agit donc ni d'extraire des candidats termes, ils sont connus des experts : « chiton », « chiton arrhaphos », « chlamys », « exomis », « himation », « zeira », etc., ni de rechercher les différents sens des termes et leurs variations en discours. L'expert s'intéresse ici aux définitions de choses, c'est-à-dire aux définitions des concepts, dénotés par les termes, exprimées à l'aide de connaissances extralinguistiques qui ne varient pas en fonction des discours auxquels ces concepts peuvent donner lieu : un « vêtement d'une pièce avec manches » reste un « vêtement d'une pièce avec manches » même si le terme qui lui est associé prend un sens (signifié) différent dans un contexte particulier. On distingue donc le signifié, construit en discours, du concept par nature extralinguistique, distinction d'autant plus nécessaire que, comme le rappelle F. Rastier [69], «le lexique des langues ne reflète pas la conception scientifique du monde ».

Les experts suivent ainsi une démarche terminologique, au sens de la Terminologie prise comme discipline scientifique comme nous le verrons au chapitre suivant, qui distingue la définition terminologique ${ }^{(5)}$, qui est une définition de chose, c'est-à-dire une définition de la chose (concept) dénotée par le terme, de la définition lexicographique ${ }^{(6)}$, qui est une définition de mot, c'est-à-dire une définition exprimant le sens des emplois du terme en discours.

L'objectif est également de voir en quoi la Terminologie dite «classique », celle de Wüster et de l'ISO, et en quoi l'Intelligence Artificielle, à travers ses théories et modèles de représentation de connaissances descriptives portant sur les objets du

(5) « De la définition ontologique d'Aristote à la définition constructive (fonctionnelle) de la mathématique, on reste sur le même terrain : on ne définit, non pas des mots, mais des termes organisés en systèmes structurés et reflétant une organisation conceptuelle, formelle (consistante), qu'elle soit considérée ou non comme reflétant les structures mêmes de l'être. » [70].

(6) «Quant à la définition lexicographique, elle concerne seulement les signes d'une langue : elle explicite des signifiés tout en essayant de distinguer, non des concepts et des classes de choses, mais des sens et des classes d'usage (d'emplois) des signes. » (ibid). 
monde, peuvent apporter aux Humanités Numériques dans le cadre de la construction de dictionnaires de choses et dans le respect des modes de pensée des experts. En effet, il est important, non pas de contraindre les experts du domaine à s'adapter aux outils informatiques mis à leur disposition, mais, au contraire, de proposer des méthodes et outils conformes aux modes de pensée et de travail des experts. Ainsi, si les experts ne s'accordent pas nécessairement sur la façon de conceptualiser, d'organiser, et de classer les objets, tel expert considérant par exemple que la caractéristique d'être un vêtement de dessous n'est pas important alors que ce sera le cas pour un autre expert : «Rejetons, comme nous l'avons dit précédemment, une classification générale à la fois inutile et peu exacte que préconisèrent jadis certains auteurs, classification qui tendrait à séparer les vêtements grecs en deux catégories, ceux de dessous et de ceux de dessus » [58], tous, néanmoins, s'accordent à penser que les termes font référence à des regroupements d'objets «semblables », que la solution réside dans la modélisation des connaissances du domaine structurant ces objets, et qu'il existe des «primitives de connaissance », des caractéristiques essentielles ${ }^{(7)}$, à partir desquelles se définissent et se structurent les concepts. Si ce principe de caractéristique essentielle, sur lequel se bâtissent et se structurent les connaissances conceptuelles du domaine, est simple à comprendre, la gestion de l'ensemble des caractéristiques essentielles et de leurs combinaisons possibles est loin de l'être, et peut difficilement être faite sans une aide informatique $^{(8)}$.

Enfin, le patrimoine culturel est un domaine où les technologies sémantiques devraient prendre une part de plus en plus importante. Si l'archéologie s'est toujours appuyée sur les outils numériques, et si la philologie classique et les domaines connexes ont été le fer de lance de grands projets de numérisation, ces domaines ont été assez lents à adopter une approche formelle et normalisée de leurs terminologies s'appuyant sur des standards du W3C à des fins d'interopérabilité : «Since many scholars question the utility of standardized terminology, the formalization of the major terminological systems in the sector is still poor, but this may be overcome by a gradual transfer of know-how and better appreciation of the specifics of cultural conceptualization by ontology engineers » [26]. Cela est probablement dû au fait que ces disciplines travaillent à l'intérieur de frontières linguistiques et culturelles spécifiques et pour lesquelles les connaissances et les modes de pensée de l'expert se prêteraient mal à une formalisation, une normalisation et une modélisation informatique.

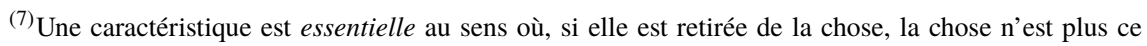
qu'elle est. Par exemple la caractéristique essentielle «avec manches » pour le chiton cheiridotos.

${ }^{(8)}$ Pour exemple, 10 couples composés chacun de deux caractéristiques essentielles exclusives entre elles («avec coutures », « sans coutures ») permettent de définir $1024\left(2^{10}\right)$ concepts terminaux (arbre de Porphyre complètement développé). Si l'introduction de dépendances entre caractéristiques réduit ce nombre ( avec une attache » n'a de sens que si le vêtement est porté «attaché » et non «enroulé autour du corps »), il augmente d'autant la charge cognitive de l'expert, rendant encore plus indispensable l'utilisation d'une méthode outillée.
} 


\subsection{ResSOURCES UTILISÉES}

Dans le cadre de notre étude, nous nous sommes particulièrement intéressés aux dictionnaires de choses. Citons pour exemple le dictionnaire accessible en ligne du Centre de Recherche d'Art Classique (Classical Art Research Centre ${ }^{(9)}$ ) dont le cœur repose sur les archives Beazley et qui contient une des plus grandes collections d'images de poteries anciennes peintes. Pour un terme donné, par exemple «chiton », le système propose une définition de chose et une représentation du vêtement (figure 2.1). Les dictionnaires également consultés ont été, pour le grec ancien, le dictionnaire standard Greek-English Lexicon de Liddell, Scott, et Jones (LSJ) [55] ${ }^{(10)}$ accessible en ligne; le dictionnaire encyclopédique de W. Smith et al. [85] accessible à partir de Perseus 4.0 $0^{(11)}$, ainsi que le Dictionnaire Grec-Français Bailly (2000), le Dictionnaire des Antiquités Grecques et Romaines (DAGR) édité par Charles-Victor Daremberg et Edmond Saglio [24] également accessible en ligne, et le glossaire dédié aux vêtements grecs et romains [20]. Cependant, espérer extraire une «conceptualisation» du domaine à partir des entrées d'un dictionnaire, à moins que les définitions soient aristotéliciennes [68], [4], [43] [82] semble difficile comme l'ont montré Ide et Veronis [45] (cf. [29]). Les dictionnaires historiques bilingues (ou monolingue) de Grec ancien que nous connaissons ne font pas exception.

\section{CLASSICAL ART RESEARCH CENTRE}

\begin{tabular}{|l|l|l|l|l|l|l|l|l|l} 
Home & Beazley Archive & Pottery database & Gem research & Terracottas & Other databases & Events & Resources & Publications & People
\end{tabular}

\begin{tabular}{l}
\hline Resources \\
\hline Databases \\
\hline Beazley Archive Contents \\
\hline Beazley Notebooks \\
\hline Library Resources \\
\hline Introduction to Greek \\
pottery \\
\hline Greek Architectural \\
Terracottas \\
\hline Sir John Boardman and \\
engraved gems \\
\hline Dictionary \\
\hline Bibliographies \\
\hline Podcasts \\
\hline \\
\hline
\end{tabular}

Use of cookies on this site

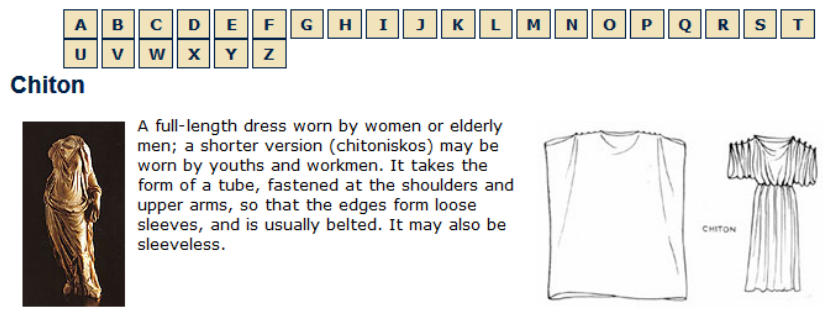

A full-length dress worn by women or elderly An: a shorter version (chitoniskos) may be rn by youths and workmen. It takes the tube, fastened at the shoulders and seeves, and is usually belted. It may also be sleeveless.

Figure 2.1. L'entrée « Chiton» dans le dictionnaire du Classical Art Research Center

\footnotetext{
${ }^{(9)}$ https://www . beazley.ox.ac.uk/index.htm

${ }^{(10)}$ http://stephanus.tlg.uci.edu/lsj

${ }^{(11)}$ http: //www . perseus. tufts . edu/hopper/collections
} 
Nous avons porté une attention toute particulière aux collections d'objets, sculptures, bas-reliefs, peintures, vases, pièces de monnaie, représentant des vêtements de la Grèce antique, accessibles à travers des sites comme celui de Harvard Art Museums ${ }^{(12)}$, de Arachne ${ }^{(13)}$ donnant accès à la base de données centrale des objets du German Archaeological Institute (DAI) et du Archaeological Institute de l'Université de Cologne, du Fine Arts Boston Museum ${ }^{(14)}$, ou tout simplement à partir de Europeana ${ }^{(15)}$, le plus grand portail multimédia ouvert qui existe dans l'espace publique numérique donnant accès aux données des institutions culturelles européennes. Nous avons également consulté des thesaurus, sources d'informations pouvant être utiles à travers l'organisation hiérarchique de leurs descripteurs traduisant une certaine classification des objets du monde, par exemple le thésaurus AAT (Art \& Architecture Thesaurus) ${ }^{(16)}$ de la fondation Getty; ainsi que certains textes accessibles en ligne provenant principalement des bibliothèques numériques Perseus 4.0 de l'Université Tufts ([23]; concernant la nouvelle version de Perseus voir [22]) et TLG-Thesaurus Linguae Graecae ${ }^{(17)}$ en ligne de l'Université de Californie à Irvine. Par contre, les réseaux sémantiques WordNet ${ }^{(18)}$ et BabelNet ${ }^{(19)}$ n'ont pas été exploités en raison d'informations qui restent, vu notre objectif et notre domaine, trop lacunaires ou sans application directe.

\section{La Terminologie}

\subsection{Dimension linguistiQue ET Dimension CONCEPTUELLE}

La Terminologie ${ }^{(20)}$ est à la fois une «science étudiant la structure, la formation, le développement, l'usage et la gestion des terminologies dans différents domaines » et le résultat de son application à un domaine sous la forme d'un « ensemble des désignations appartenant à une langue de spécialité » [46]. En posant, à l'instar des normes ISO, qu'un terme est une « désignation verbale d'un concept général » (ISO 1087-1), on accepte de facto que la Terminologie a une double dimension, linguistique et conceptuelle ${ }^{(21)}$. On s'intéresse, dans le cadre de cet article, uniquement aux termes qui sont des noms d'objets (substantifs), à l'exclusion des autres catégories de termes,

${ }^{(12)}$ https://www.harvardartmuseums . org/collections

${ }^{(13)}$ https://arachne.uni-koeln.de/drupal/

${ }^{(14)}$ https://www.mfa.org/

${ }^{(15)}$ https://www. europeana.eu/portal/fr

${ }^{(16)}$ The Getty Research Institute. http://www.getty.edu/research/tools/vocabularies/aat/

${ }^{(17)} \mathrm{TLG}=$ Thesaurus Linguae Graecae. (2001). A Digital Library of Greek Literature. University of California, Irvine. http://stephanus.tlg.uci.edu

${ }^{(18)}$ https://wordnet.princeton.edu/

${ }^{(19)}$ https://babelnet.org/

${ }^{(20)}$ Nous noterons «Terminologie » avec une majuscule pour désigner la discipline, avec une minuscule pour désigner un ensemble de termes dans un domaine donné.

(21) Afin de bien distinguer les deux dimensions, linguistique et conceptuelle, qui composent toute terminologie, nous utiliserons les conventions de notation suivantes : les termes sont notés entre guillemets et commencent par une minuscule, par exemple «exomide » dénotant un type de vêtements particulier, les concepts entre chevrons commençant par une majuscule, par exemple <Vêtement pour homme sans manche>, les caractéristiques essentielles entre barres verticales, /avec manches/ et /pour homme/ en sont des exemples, et la référence à des objets en italique : chiton, exomis, chlamys, etc. 
et uniquement à leur définition terminologique (définition de chose), c'est-à-dire à leur définition au regard d'une modélisation des connaissances conceptuelles du domaine, indépendamment du sens que ces termes peuvent prendre en discours (i.e. leurs signifiés). Cette approche de la Terminologie nécessite de préciser d'une part la théorie $d u$ concept sur laquelle elle repose, et d'autre part le langage utilisé pour définir le modèle conceptuel.

\subsection{THÉORIE DU CONCEPT}

La notion de concept, connaissance portant sur une pluralité de choses, vise à appréhender le « réel » et à organiser les objets qui le peuplent. Il existe pour cela différentes façons de procéder, selon la nature des objets, leur structure, leur état, leur fonction, et donc autant de définitions du concept possibles, ce qui peut constituer un obstacle à une collaboration entre experts du domaine et ingénieurs de la connaissance : «[...] we found that a major obstacle to introducing advanced computer science methods in the sector is a general underestimation of the complexity of cultural heritage conceptualization by the IT experts, which is equalled by the inability of domain experts to describe their conceptualizations in conscious, objective terms » [26]. La Terminologie, quant à elle, définit le concept comme une unité de connaissance créée par une combinaison unique de caractéristiques essentielles ${ }^{(22)}$. De plus, «Les concepts n'existent pas en tant qu'unités de connaissance isolées mais sont toujours en relation les uns par rapport aux autres. » [47]. Ils se structurent en un système selon différents types de relations, génériques, partitives, associatives, qui participent à leur définition.

\subsection{LANGAGES DE REPRÉSENTATION}

En tant que «unités de connaissance », les concepts sont de nature extralinguistique, au sens où ils ne relèvent pas de la langue naturelle. Néanmoins, un langage est nécessaire pour les définir : il n'y a pas de connaissances sans langage. Si une définition du concept en langue naturelle sera in fine nécessaire, ce n'est pas le langage $^{(23)}$ le plus adapté pour cela : ce n'est pas son rôle ${ }^{(24)}$. Nous avons besoin d'un langage dédié à la construction de systèmes conceptuels. C'est-à-dire un langage qui supporte les principes de la théorie du concept propre à la Terminologie. Ces langages sont plus ou moins formels selon les objectifs visés et/ou la phase de construction du système notionnel. On peut distinguer différentes familles de langages [80]. Les langages graphiques ${ }^{(25)}$ qui constituent une aide à la construction et à la compréhension du système conceptuel, les langages de représentation des connaissances issus de

\footnotetext{
${ }^{(22)}$ Voir la note 7 précédente.

${ }^{(23)}$ On distingue «langue » désignant une langue naturelle ( «natural language » en anglais) de « langage» («artificial language ») désignant un système artificiel de signes.

${ }^{(24)}$ La langue naturelle «parle» du monde, reste un discours sur le monde, en cela elle peut être qualifiée de méta langue. Contrairement aux « langages scientifiques » dont c'est la finalité, elle ne construit pas une représentation du monde qui peut être manipulable à des fins de calcul (par un ordinateur lorsque ces langages lui sont compréhensibles).

${ }^{(25)}$ La norme (ISO 24156, 2014) [48] propose une représentation graphique du système conceptuel basée sur la notation de la norme UML (Unified Modeling Language). Il est également possible d'utiliser
} 
l'Intelligence Artificielle ${ }^{(26)}$, ceux du W3C tels que SKOS ${ }^{(27)}$ et $\operatorname{RDFS}^{(28)}$, et enfin les langages de type logique qui jouent un rôle particulièrement important en raison de leur formalisme universel. Citons comme exemple de ces derniers langages les logiques de description [5]. Basées sur les notions d'individu, de classe (ensemble d'individus) et de rôle (relation binaire entre individus), elles constituent un moyen puissant de représentation des connaissances proposant deux opérations de classification : une classification hiérarchique des classes et une classification des individus dans les classes correspondantes. OWL ${ }^{(29)}$ est un langage du W3C construit sur RDF basé sur les logiques de description.

\subsection{Quel LANGAge ChOISIR}

Le choix du langage de représentation des concepts, qu'il soit formel ou non, n'est pas anodin. Il dépend de différents paramètres, par exemple de la phase de construction du système conceptuel dans laquelle on se trouve. Ainsi, «dessiner» un graphe de concepts peut être utile pour démarrer un projet. Il dépend surtout des objectifs visés et des propriétés que l'on souhaite garantir. Le choix doit être également fait en gardant à l'esprit qu'il n'est pas sans conséquence sur la façon dont on perçoit le monde, et donc sur la conceptualisation elle-même : on pense en fonction d'un langage et des principes épistémologiques qu'il véhicule, notre «vision » du monde se réduit à ce qu'on en peut dire dans un langage donné. Il n'est également pas sans conséquence sur les définitions en langue naturelle des termes si l'on souhaite les justifier au regard des définitions formelles des concepts dénotés. Ainsi la définition aristotélicienne du terme en genre prochain et différence(s) spécifique(s) repose sur la notion de concept défini comme une spécialisation d'un concept plus générique augmentée d'une ou plusieurs caractéristiques essentielles. Il est à souligner que la notion de caractéristique essentielle sur laquelle repose la Terminologie, mais aussi la façon de penser et de travailler des experts, n'existe pas en tant que telle dans les langages à base de schémas et les langages logiques. Elle peut bien évidemment être traduite sous la forme d'un attribut à valeur booléenne pour les langages à base de schémas, ou, pour les langages de description, sous la forme d'individus, de classes disjointes, de restrictions de rôles, mais elle n'existe pas en tant que catégorie de pensée supportée par ces langages.

\footnotetext{
des éditeurs tels que CmapTools, logiciel libre d'édition de cartes conceptuelles issu des travaux de recherche de l'institut IHMC (Florida Institute for Human \& Machine Cognition) : https: //cmap . ihmc .us/

${ }^{(26)}$ Ces langages ont une longue histoire $[60,14,73,95,33]$

${ }^{(27)}$ Simple Knowledge Organization System «A common data model for sharing and linking knowledge organization systems via the Web» https://www.w3.org/TR/2009/ REC-skos-reference-20090818/

${ }^{(28)}$ Resource Description Framework Schema «A data-modelling vocabulary for RDF data $»$ https : //www.w3.org/TR/rdf-schema/

(29) « a Semantic Web language designed to represent rich and complex knowledge about things, groups of things, and relations between things » : https://www.w3.org/OWL/
} 


\section{L'ONTOLOGIE}

L'utilisation de langages formels compréhensibles par un ordinateur pour la représentation du modèle conceptuel conduit à la notion d'ontologie ${ }^{(30)}$ au sens de l'Ingénierie des Connaissances [91, 42]. Définie initialement comme « an explicit specification of a conceptualization » [40], elle a donné lieu à différentes définitions ${ }^{(31)}$ que détaille l'article «What Is an Ontology?» [42] ainsi qu'à de nombreux travaux [86]. Issue de la philosophie, l'Ontologie vise à comprendre, décrire et organiser les objets qui peuplent une réalité. En représentation des connaissances, elle prend la forme d'une conceptualisation $^{(32)}$ d'un domaine partagée par une communauté de pratique. Construite pour un objectif donné, elle est définie dans un langage qui se veut compréhensible à la fois par des êtres humains et des machines.

L'Ontologie constitue une des voies les plus prometteuses pour la Terminologie [76]. En proposant une représentation formelle et computationnelle de son système conceptuel, elle permet l'écriture de définitions objectives au sens où leur interprétation est régie par la théorie, indépendamment de toute interprétation individuelle, et ouvre la voie à l'opérationnalisation des terminologies à des fins de traitement de l'information comme les moteurs de recherche sémantique, la gestion de contenus multilingues, le calcul automatique d'équivalents terminologiques, etc. Elle intervient également fortement dans les Humanités Numériques où le terme de «ontologie » désigne soit la conceptualisation d'un domaine (on parlera d'ontologies de domaine), soit le langage utilisé pour décrire les objets du monde, ce qui peut entraîner une certaine confusion : «[...] formal ontologies were introduced to computer science to describe common conceptualization behind multiple schemata, and not to become a synonym of schemata » [26]. Ces deux approches sont indissociables de l'ouverture sur le web des Humanités numériques : «Semantic interoperability of Digital Libraries, Libraryand Collection Management Systems requires compatibility of both the employed Knowledge Organization Systems (KOS; e.g. classification systems, terminologies, authority files, gazetteers) and of the employed data and metadata schemata » [27]. En dehors de l'utilisation d'ontologies comme vocabulaires de description des objets et des faits culturels, c'est bien évidemment comme conceptualisation d'un domaine que l'ontologie nous intéresse ici. Les ontologies sont en général spécifiques, que ce soit à un sujet, une collection, une période donnée. Une diversité que l'on retrouve au sein de mêmes domaines et qui soulève, dans le cadre de leur interopérabilité, le problème de leurs mises en correspondance (alignement). Mais c'est principalement à travers les thésaurus, dans l'organisation hiérarchique de leurs descripteurs ${ }^{(33)}$, qu'interviennent

\footnotetext{
${ }^{(30)}$ Nous n'aborderons pas ici l'Ontologie dans son acception philosophique, dont les apports pour la représentation des connaissances et la Terminologie ne peuvent néanmoins être négligés.

${ }^{(31)}$ Dont celle qui définit une ontologie comme «[...] a vocabulary of terms and some specification of their meaning » [91].

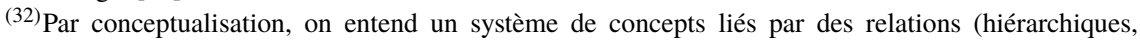
partitives, associatives).

${ }^{(33)}$ En considérant les descripteurs comme des termes ou des concepts structurés selon des relatons hiérarchiques, il est possible de «traduire » des thesaurus en ontologies : « The full AAT hierarchy was converted into a hierarchy of concepts, where each concept has a label slot corresponding with the main term in AAT and a synonyms slot where alternate terms are represented » [93].
} 
les ontologies pour l'Héritage Culturel. Le thésaurus AAT introduit au paragraphe 2 est un exemple d'ontologie qui couvre un large champ de l'Héritage Culturel. Les thésaurus PACTOLS du programme Frantiq, conçus à l'origine pour indexer des ressources sur les civilisations de l'Antiquité, couvrent aujourd' hui l'archéologie de la Préhistoire à la Seconde Guerre mondiale ${ }^{(34)}$. Les projets européens FP7 Linked Heritage ${ }^{(35)}$ et AthenaPlus $^{(36)}$ sont deux exemples de projets dans le domaine culturel en lien avec Europeana où ces notions ont été mises en œuvre.

\section{Ontoterminologie}

\subsection{DÉfinition}

Aujourd'hui, l'utilisation d'un langage formel compréhensible par un ordinateur pour la définition des concepts semble inévitable si on souhaite vérifier un certain nombre de propriétés portant tant sur la définition des concepts (objectivité, cohérence, complétude) $)^{(37)}$ que sur le système conceptuel lui-même (propriétés des relations hiérarchiques et associatives), ou si on souhaite tout simplement opérationnaliser les terminologies. C'est la raison pour laquelle nous nous sommes intéressés à la notion d'ontologie au sens de l'ingénierie des connaissances. Associée à la terminologie, elle conduit à la notion d'ontoterminologie, terminologie dont le système conceptuel est une ontologie formelle. Introduite en 2007 et issue de travaux menés en ingénierie des connaissances et en terminologie [77], l'ontoterminologie explicite la double dimension de la Terminologie en deux systèmes différents mais liés entre eux. Ainsi, les termes et les noms de concepts (identifiants) ne se confondent pas, tout comme les signifiés et les concepts. Même si elles entretiennent des liens forts, ces notions ne relèvent pas des mêmes systèmes sémiotiques. De même, la définition du terme en langue naturelle, qu'elle traduise l'usage du mot (définition de mot) ou ce qu'il désigne (définition de chose), ne se confond pas avec la définition formelle du concept. Une telle approche permet de normaliser la seule chose qui peut l'être, à savoir les connaissances du domaine, et de préserver ce qui doit l'être, à savoir la diversité langagière.

\subsection{TEDI}

La construction d'ontologies est une tâche difficile [44] qui requiert des environnements dédiés. Protégé(38), supporté par une forte communauté d'utilisateurs, est le plus connu d'entre eux. Développé par le Centre de Recherche en Informatique Biomédical de l'Université de Stanford (Stanford Center for Biomedical Informatics Research, Stanford University School of Medicine), Protégé est un logiciel libre d'édition d'ontologies basées sur les logiques de description et supportant le langage OWL, le langage

\footnotetext{
${ }^{(34)}$ https: //multimedia.inrap.fr/Ressources-documentaires/

Produits-documentaires/p-17078-Thesaurus-PACTOLS.htm

${ }^{(35)}$ http: //www. linkedheritage.eu/

${ }^{(36)}$ http: //www . athenaplus.eu/

${ }^{(37)} \mathrm{Au}$ regard de la théorie du concept choisie.

(38) urlhttps ://protege.stanford.edu/
} 
standard du W3C pour les ontologies. Cependant, notre choix ne s'est pas porté sur l'environnement Protégé dont la maîtrise demande des compétences spécifiques : «As the group that developed Protégé, the most widely used ontology editor, we are keenly aware of how difficult the users perceive this task to be. » [44] Les experts ne sont pas nécessairement rompus à la définition de concepts en termes de rôles et de restrictions de rôles [28]. Basé sur une approche extensionnelle où la notion de concept est remplacée par celle de classe d'individus liés entre eux par des relations [5] Protégé ne permet pas de prendre en compte directement la notion de caractéristique essentielle qui nous intéresse ici, elle doit être traduite d'une façon ou d'une autre. Enfin il n'est pas possible de guider la construction de l'ontologie en tenant compte de la dimension linguistique de la terminologie qui se réduit essentiellement à des étiquettes sur des concepts et à des notes, même si elle peut être complétée par une «lexicalisation » de l'ontologie [16, 1].

Il existe peu d'environnements permettant d'éditer à la fois les dimensions linguistique et ontologique [12], et des environnements comme Terminae [89] centrés sur la modélisation du sens en discours, ne correspondent pas à nos besoins ${ }^{(39)}$, qui, rappelons-le, se focalisent sur la modélisation des connaissances indépendamment des discours auxquels elles peuvent donner lieu. Dans le cadre de projets relevant des Humanités Numériques nous pouvons citer $\operatorname{ELEON}^{(40)}$ [13] et, parmi les éditeurs les plus récents, $\mathrm{LexO}^{(41)}$ [11], et TEDI ${ }^{(42)}$ [64]. Le choix s'est porté sur TEDI (ontoTerminology EDItor), environnement logiciel dédié à la construction d'ontoterminologies multilingues. Contrairement à Protégé, TEDI n'a pas une visée universelle dans la construction d'ontologies. Il repose sur une théorie du concept dédiée à la Terminologie, la C.T.T. (Concept Theory for Terminology) et propose une méthodologie outillée de construction d'ontoterminologies respectant la démarche de l'expert.

\subsubsection{La C.T.T.}

La C.T.T. (Concept Theory for Terminology) [81] est une théorie du concept dédiée à la Terminologie prenant en compte la démarche et la façon de penser des experts. Elle tient compte des critiques émises à l'encontre de la Terminologie dite «classique » [79] telle qu'elle est prônée par la Théorie Générale de la Terminologie [30] et l'ISO [46, 47], mais aussi des propositions faites dans le cadre de la révision en cours de la norme ISO $1087-1^{(43)}$. Elle tient compte également des acquis et des progrès réalisés dans des disciplines connexes à la Terminologie, que ce soit en linguistique,

${ }^{(39)}$ Terminae est fondé sur la notion de termino-concept défini comme un « terme désambigüisé dont le sens est défini par son usage dans le corpus » [89]

${ }^{(40)}$ ELEON (Editor for Linguistically Enriched Ontologies) est un éditeur qui permet d'enrichir des ontologies OWL par des annotations linguistiques et de générer des descriptions textuelles des objets : http ://users.iit.demokritos.gr/ eleon/ELEON.html

${ }^{(41)}$ Basé sur Lemon, LexO est un éditeur collaboratif de ressources lexicales, terminologiques et ontologiques

${ }^{(42)}$ TEDI (ontoTerminology EDItor), écrit en Smalltalk (VisualWorks 8.3), est développé par C. Roche à l'Université Savoie Mont-Blanc : http: //ontoterminology . com/

${ }^{(43)}$ C. Roche est Project Leader ISO de la norme ISO 1087-1 en cours de révision. 
en ingénierie des connaissances ou en logique, mais aussi des enseignements tirés de l'opérationnalisation des terminologies à des fins de traitement de l'information. La C.T.T. repose sur les notions de caractéristique essentielle exprimant la nature des choses (caractéristique non valuée) et de caractéristique descriptive décrivant les états possibles d'une chose (caractéristique valuée) comme la couleur ${ }^{(44)}$, le type de fibre. Elle distingue la notion de concept, portant sur des objets de même nature, c'est-à-dire partageant les mêmes caractéristiques essentielles, de la notion de classe regroupant des objets pouvant être de nature différente mais vérifiant une même propriété, par exemple la classe des vêtements en lin regroupant aussi bien des chlamydes que des chitons. La notion de relation est importante et donne lieu à une typologie fine en particulier en ce qui concerne la relation partitive.

\subsubsection{Dimension conceptuelle de TEDI}

TEDI propose différents éditeurs dédiés à la dimension conceptuelle : éditeurs de concepts, d'objets, d'axes d'analyse (ensembles de caractéristiques essentielles exclusives entre elles nommées différences dans TEDI), de caractéristiques descriptives (nommées attributs), et de relations.

La définition des concepts et leur mise en relation se fait à l'aide de l'éditeur de concepts (figure 5.1) qui s'appuie sur les principes épistémologiques et les propriétés logiques de la C.T.T. pour guider l'utilisateur dans sa construction. Ainsi, seules les caractéristiques essentielles valides, au sens de la théorie, sont proposées à l'expert lors de la définition ou de la mise à jour d'un concept, ce qui constitue une aide considérable pour l'expert dans la gestion de la complexité du domaine ${ }^{(45)}$. L'héritage peut être multiple, TEDI vérifiant la compatibilité des caractéristiques héritées. à noter qu'il est possible d'illustrer concepts et objets à l'aide d'images et de vidéos.

\subsubsection{Dimension linguistique de TEDI}

L'éditeur de termes de TEDI (figure 5.2) permet de compléter les informations relatives aux termes : définition en langue naturelle (un «pattern » de définition du terme en langue naturelle est généré sur la base de la définition formelle du concept dénoté par le terme, le pattern commence par l'hyperonyme terminologique direct, lorsqu'il existe, suivi des caractéristiques essentielles du concept), statut du terme (préféré, alternatif, toléré, déconseillé, obsolète), catégorie grammaticale, variations terminologiques et formes fléchies, ajout de contextes et de notes.

${ }^{(44)}$ La couleur peut être dans certains cas une caractéristique essentielle, distinguant par exemple des classes sociales. Ainsi, dans la Rome antique les patriciens portaient des tuniques blanches, les sénateurs une tunique avec des rayures violettes appelées la tunica laticlavi, les empereurs la trabea, toge entièrement violette.

${ }^{(45)}$ Voir la note 8 sur les arbres de Porphyre. 


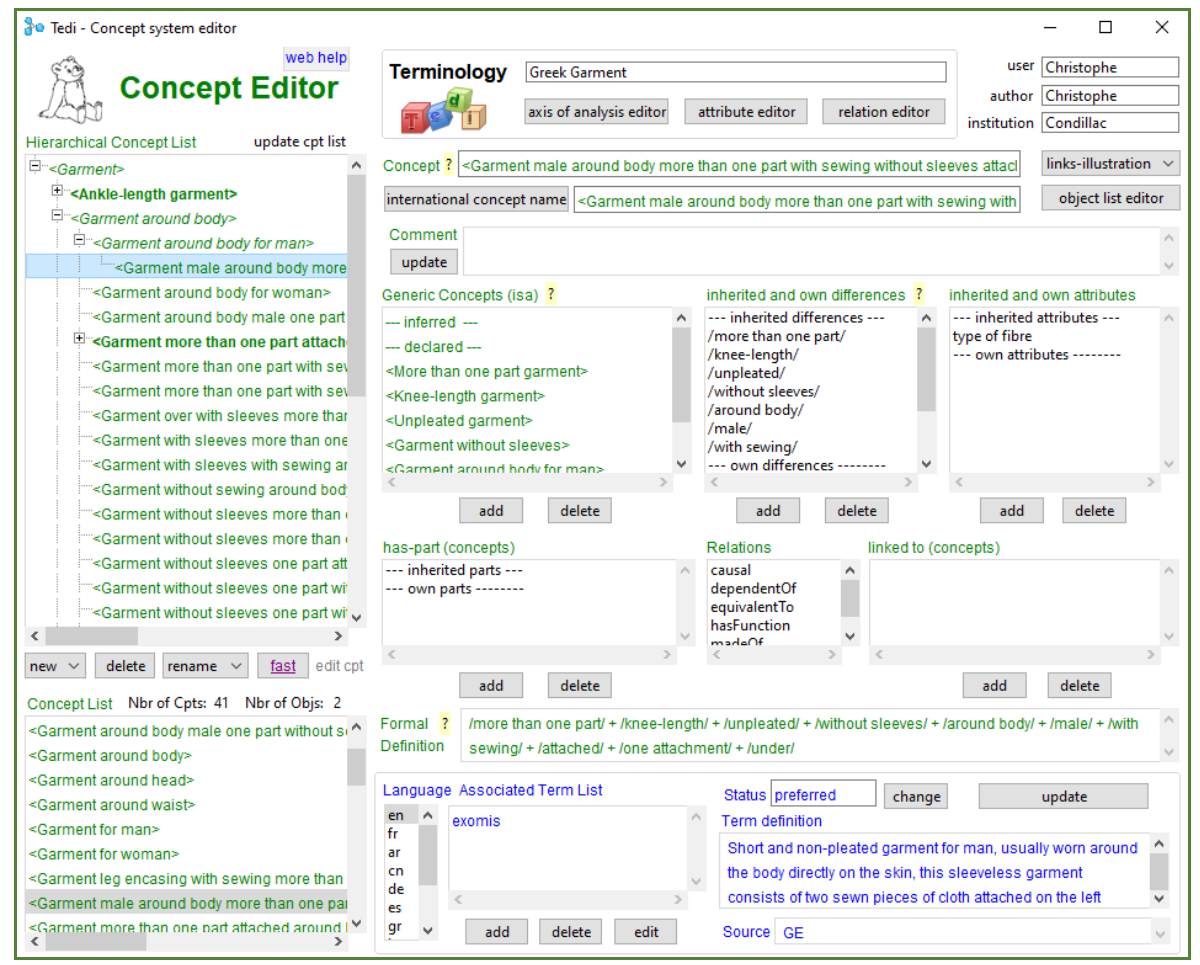

FIgURE 5.1. L'éditeur de concepts de TEDI

TEDI calcule automatiquement les synonymes terminologiques, hyperonymes et hyponymes terminologiques $^{(46)}$, ainsi que les équivalents terminologiques directs (termes dénotant le même concept dans une autre langue) et les équivalents terminologiques hyperonymes et hyponymes (termes dénotant un concept générique versus spécifique dans une autre langue).

\subsubsection{Export}

Le partage et l'interopérabilité des données reposent sur la mise en œuvre des ontoterminologies dans le respect des normes ISO pour leur construction et des standards $\mathrm{du}$ W3C pour leur ouverture sur le Web. Les ontoterminologies sont exportées dans

${ }^{(46)}$ Pour une langue donnée, deux termes sont des synonymes terminologiques si et seulement s'ils dénotent le même concept (à distinguer des synonymes discursifs, communément appelés synonymes, déterminés sur la base des signifiés des termes construits en discours). De façon similaire, en distinguant la relation conceptuelle générique is- $a$, liant des concepts, de la relation d'hyperonymie terminologique liant des termes (à distinguer de la relation d'hyperonymie discursive communément appelée hyperonymie), le terme « $\mathrm{t}_{\mathrm{a}}$ » est un hyperonyme terminologique (respectivement hyponyme terminologique) du terme « $\mathrm{t}_{\mathrm{b}}$ » $\mathrm{si}$ le concept dénoté par « $\mathrm{t}_{\mathrm{a}}$ » est un concept générique (respectivement concept spécifique) du concept dénoté par $\ll \mathrm{t}_{\mathrm{b}} »$. 


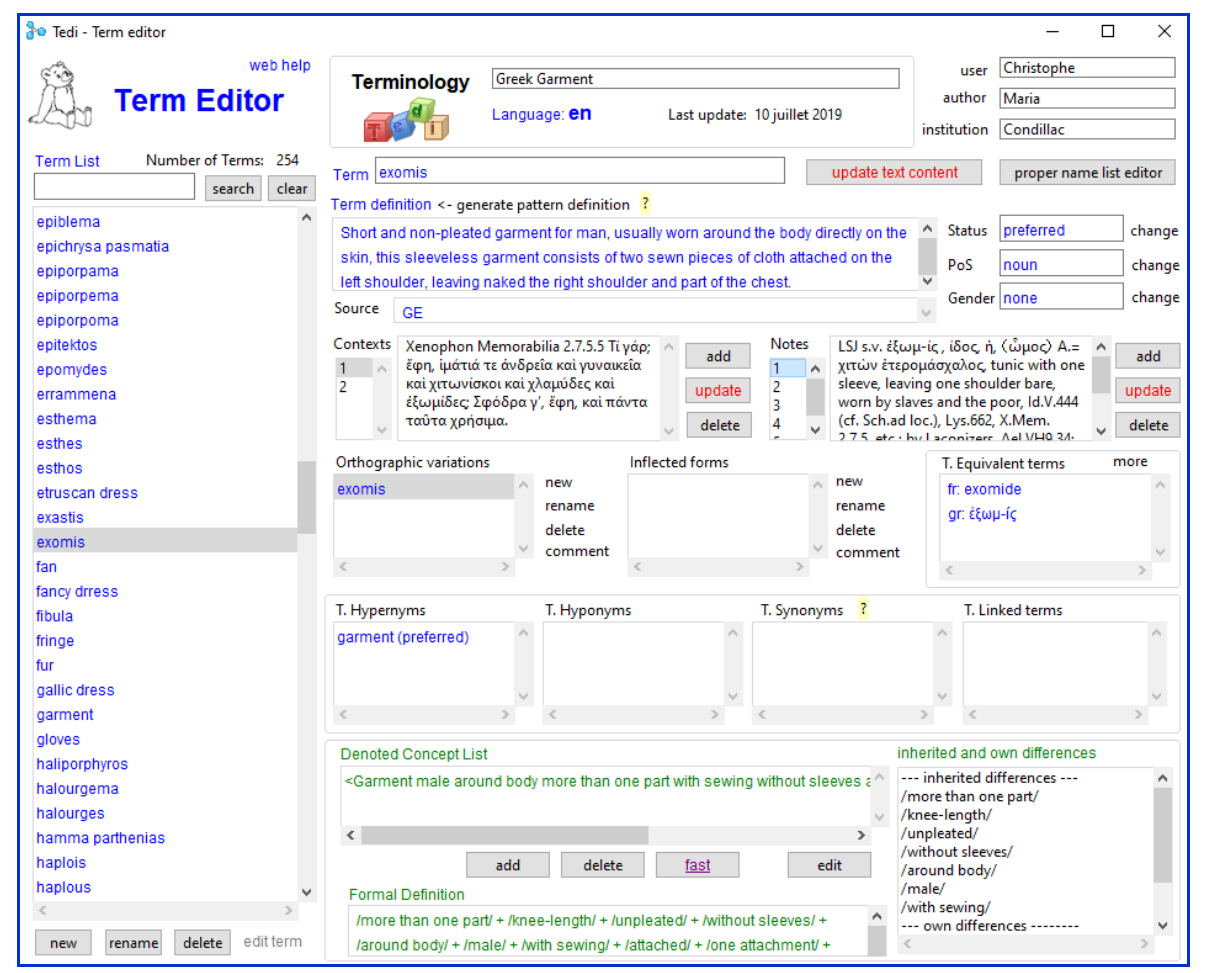

Figure 5.2. L'éditeur de termes de TEDI appliqué à l' « exomide »

différents formats : JSON, RDF/OWL, CSV et HTML, les formats SKOS, OntoLexLemon [59] et TEI-Lex0 [9] sont en cours de prise en compte. Ces formats d'échange ne sont pas équivalents et il peut y avoir perte d'informations lors du processus de traduction dans ces formats. Nous verrons dans le chapitre suivant un exemple d'export sous la forme d'un dictionnaire électronique (figure 5.3). Citons pour exemple la traduction en OWL. TEDI traduit les caractéristiques essentielles d'un même axe d'analyse (exclusives entre elles) sous la forme de classes disjointes, les concepts comme autant de sous-classes des classes (intersection) correspondant aux caractéristiques essentielles qui définissent le concept, les relations comme des «object properties » et les caractéristiques descriptives, selon le type de leurs valeurs, comme des « object properties » ou des « data properties ». L'association d'une URI à chaque information (concept, objet, terme, relation, etc.) permet d'ouvrir les ontoterminologies de TEDI au LOD (Linked and Open Data). 


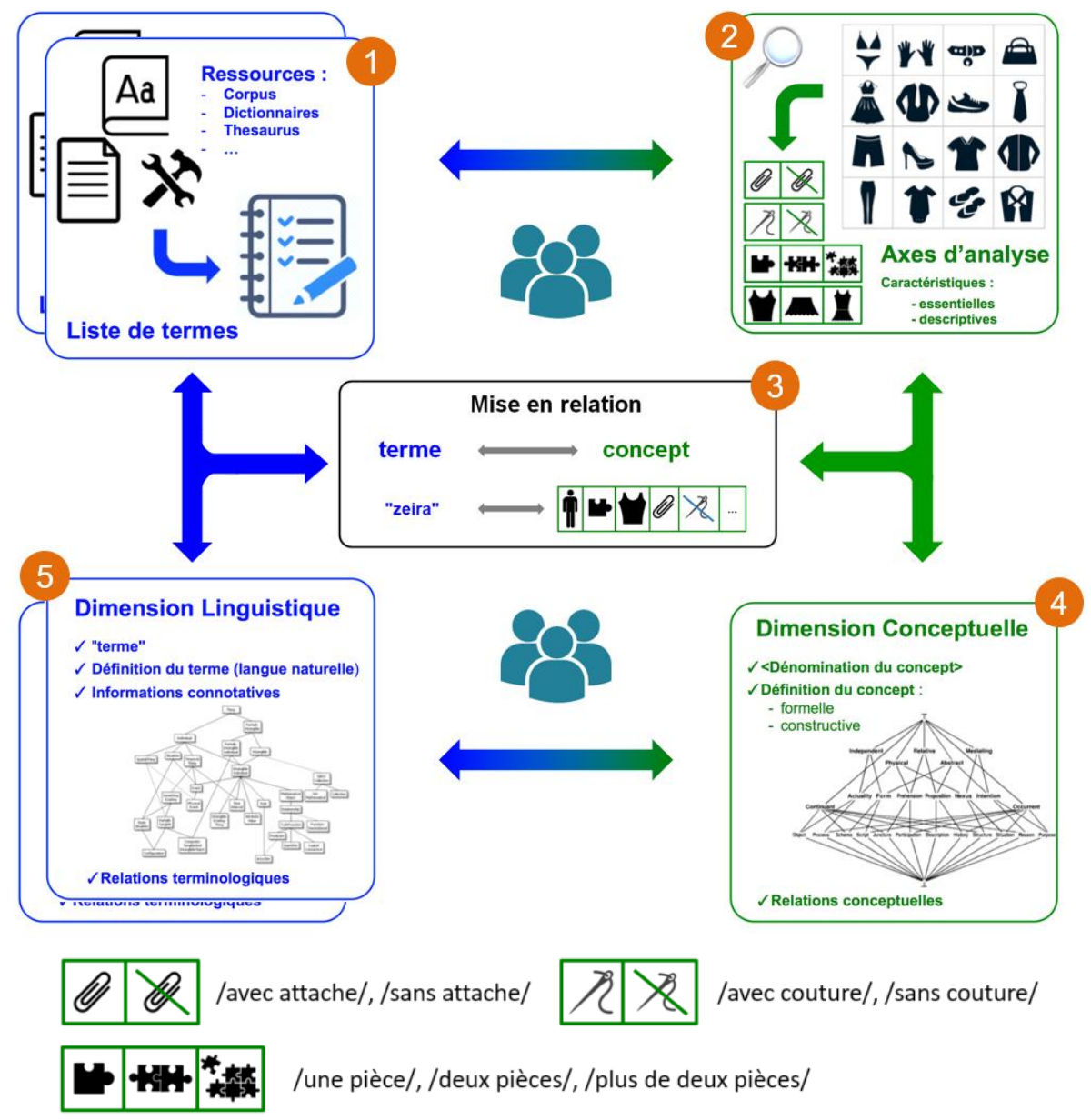

Figure 5.3. Méthodologie de construction d'ontoterminologies multilingues

\section{L'ONTOTERMinologie des VÊTEMENTS de LA GrÈCE ANTIQUe}

Il existe peu de travaux dédiés à une modélisation ontologique de terminologies dans le domaine des vêtements [2]. L'ontologie VetiVoc, « a modular ontology for the Fashion, Textile, and Clothing domain » [2], est un exemple intéressant qui, s'il illustre bien tout le pouvoir d'expression des logiques de description, en montre aussi la complexité pour des humanistes ${ }^{(47)}$. À notre connaissance, il n'existe pas de terminologies des vêtements de la Grèce antique dont les définitions reposent sur une modélisation

${ }^{(47)}$ VetiVoc suit une démarche similaire à ce qui est pratiqué en Terminologie dans sa recherche de caractéristiques définitoires : «col italien », « fermeture à glissière », etc., mais n'est cependant pas réutilisable pour les vêtements de la Grèce antique : la finalité, l'époque, les objets et les techniques sont trop différents. 
ontologique des connaissances du domaine sur la base de caractéristiques essentielles au cœur des définitions aristotéliciennes chères aux experts.

\subsection{UnE MÉTHOdOLOGIE SPÉCIFIQUE}

Il existe différentes méthodes de construction d'ontologies [92, 41, 21, 31], s'appuyant sur des critères qui se veulent objectifs (clarté, cohérence, extensibilité, etc.) [39], s'inspirant de différents domaines : développement de bases de connaissances [54], méthodes de génie logiciel [32], construction à partir de textes [15], approche modulaire de la conception [61], ingénierie ontologique [88], etc. Le choix d'une méthode n'est pas sans conséquence sur le résultat obtenu. Il dépend de différents critères : le domaine d'application, l'objectif visé (une terminologie pour quoi faire?), le type de terminologie (descriptive ou prescriptive), son éventuelle opérationnalisation (à des fins de traitement de l'information par exemple), la nature des connaissances mises en jeu, la disponibilité des experts, etc. On ne procède pas de la même façon si on doit fournir un lexique de termes en usage dans le domaine touristique ou définir une terminologie normative dans le domaine des accidents graves du nucléaire. De même, on ne construit pas de la même façon un système conceptuel pour un domaine où les connaissances sont clairement formalisées et pour lequel le concept peut-être le point de départ, que pour un domaine dont on ne dispose que de ressources textuelles et pour lequel c'est le terme qui est le point de départ [90]. Le choix de donner la priorité au terme ou au concept n'est pas sans conséquence sur la démarche et le résultat obtenu ${ }^{(48)}$. Ce choix est parfois conditionné par l'indisponibilité des experts qui oblige à se reporter sur les autres ressources disponibles en particulier linguistiques (textes). Les notions de Terminologie textuelle et de Terminologie conceptuelle en découlent directement.

Les problèmes que pose la construction de terminologies dans le domaine de l'Héritage Culturel et des vêtements de la Grèce antique tels qu'ils ont été exposés dans les paragraphes 1 et 2 , nous ont amenés à définir une méthodologie de construction d'ontoterminologies spécifique aux domaines de la culture matérielle [63, 64]. Elle est le résultat de travaux menés dès 2011 dans le domaine de l'Héritage Culturel à travers deux projets européens (Linked Heritage et AthenaPlus), des projets sur la peinture [72], la poterie [3], et d'une collaboration dans le domaine des objets matériels des cultures antiques initiée en 2014 avec l'Université de Copenhague dans le cadre d'un projet Marie Curie ${ }^{(49)}$ [62].

Cette méthodologie prend en compte la façon de penser et de travailler des experts. Elle s'appuie sur la notion de caractéristique essentielle sur laquelle se fonde les définitions du concept et du terme en Terminologie, et concilie, en les alternant, les

${ }^{(48)}$ Les ontologies qui en résultent se superposent rarement : «le lexique des langues ne reflète pas la conception scientifique du monde » [69], « dire n'est pas concevoir » [78].

${ }^{(49)}$ Marie Skłodowska Curie Actions Project Chlamys. The cultural biography of a garment in Hellenistic Egypt grant no. 657898 
approches textuelle et conceptuelle. Elle repose sur deux constats et un principe liant les dimensions linguistique et conceptuelle :

- le premier constat est que, s'il est possible d'identifier et de s'accorder sur les caractéristiques essentielles qui définissent les concepts : /vêtement d'une pièce/, /pour homme/, /avec attaches/, etc., il est souvent difficile de définir directement et dans un premier temps une conceptualisation organisant les objets en une hiérarchie clairement définie ${ }^{(50)}$;

- le deuxième constat est qu'il est cependant relativement facile pour les experts d'identifier les termes du domaine.

Le principe liant les deux dimensions est qu'un terme désigne un ensemble de caractéristiques essentielles suffisamment «stable» pour être nommé en langue, ces ensembles de caractéristiques définissant les concepts du domaine. On assiste ainsi à un retour de l'importance du terme dans une démarche principalement conceptuelle : la langue participe au découpage du réel en concepts, ensembles «stables » de caractéristiques, même si la conceptualisation du domaine peut amener, principalement pour des raisons d'organisation de la connaissance, à la définition de concepts sans désignation (terme) en langue.

Cette méthodologie est outillée à l'aide de TEDI. C'est-à-dire que TEDI offre un ensemble d'éditeurs et de fonctionnalités spécifiques à cette méthodologie comme l'inférence des hiérarchies possibles entre les concepts, la proposition de dénominations de concepts, à ne pas confondre avec les termes les désignant, ou la génération de patterns de définition en langue naturelle sur la base des définitions formelles des concepts. Cette méthodologie est composée de 5 tâches liées entre elles de façon non linéaire, l'expert passant de l'une à l'autre selon l'avancée de son travail.

Pour la clarté de la présentation, ces tâches sont décrites dans l'ordre de leur apparition en les illustrant avec l'exemple de l'exomide («exomide » en français, « exomis » en anglais, « $\varepsilon \xi \omega \mu i \varsigma$ » en grec ancien, et « $\varepsilon \xi \omega \mu i ́ \delta \alpha »$ en grec moderne). Une exomide est à l'origine une tunique attachée à l'épaule gauche seulement ( $\chi$ เ๘ìv

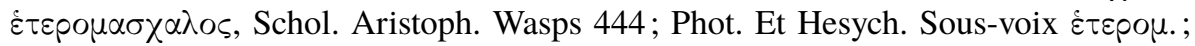
Heliod. Aethiop. 3.1; Paus. 5.16.2; cf. Plaut, Mil. 4.4, 44), laissant l'épaule droite et une partie du sein libre ${ }^{(51)}$.

\subsubsection{Tâche 1 : la sélection des termes à définir}

Éditeur de termes de TEDI : une des premières tâches de l'expert consiste à sélectionner l'ensemble des termes à définir. Rappelons que seul l'expert est habilité à dire ce qui est terme de ce qui ne l'est pas. En effet, toute unité lexicale de spécialité, c'est-à-dire une unité lexicale désignant une connaissance du domaine, n'accède pas nécessairement au rang de terme, il faut pour cela qu'elle désigne une connaissance

\footnotetext{
${ }^{(50)}$ Ces caractéristiques essentielles constituent des «primitives » de connaissance sur lesquelles les experts s'accordent. Il reste à les combiner en des ensembles, les concepts, qu'il faut nommer.

${ }^{(51)}$ http://wWw.hellenicaworld.com/Greece/WS/en/Exomis.html
} 


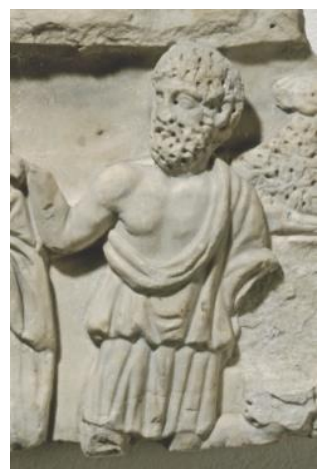

FIGURE 6.1. «Fragment vom Kasten eines einzonigen Friessarkophags » ${ }^{(52)}$

stable, non contingente. Il faut de plus distinguer les unités lexicales descriptives qui, bien qu'elles désignent des connaissances stables du domaine, ne sont pas nécessairement pour autant considérées comme des termes (au sens où elles seraient retenues comme entrées du dictionnaire terminologique) : "vêtements en lin d'une pièce sans manche » par exemple ${ }^{(53)}$. Ces termes sont créés à l'aide de l'éditeur de termes de TEDI (figure 3) pour chacune des langues prises en compte : « exomis » en anglais, « exomide » en français, « $\dot{\xi} \xi \omega \mu i \varsigma$ » en grec ancien, et « $\varepsilon \xi \omega \mu i \delta \alpha$ » en grec moderne.

\subsubsection{Tâche 2 : la définition des axes d'analyse et des caractéristiques essentielles}

Éditeur des axes d'analyse : La deuxième étape porte sur l'identification et la création des caractéristiques essentielles, ainsi que sur leur organisation en axes d'analyse. Pour cela, les experts comparent des objets qu'ils considèrent comme étant différents sur un point particulier mais essentiel. Ainsi, l'exomide est un vêtement cousu contrairement à la chlamyde, faisant de la présence ou de l'absence de coutures deux caractéristiques essentielles, exclusives l'une de l'autre (regroupées au sein du même axe d'analyse portant sur les coutures). De façon similaire, l'exomide se distingue de la candys (kandys), vêtement également cousu, par son absence de manche. La façon de porter le vêtement, le genre auquel il est dédié (homme, femme, divinité), sa longueur (non pas identifiée par une valeur, mais par la partie du corps couverte : genoux ou cheville), le nombre d'attaches, etc. sont autant caractéristiques essentielles ${ }^{(54)}$. La

${ }^{(53)}$ On ne s'intéresse pas ici à une quelconque forme de description/normalisation morpho-syntaxique des unités lexicales descriptives construites sur la base des caractéristiques définissant le type de vêtements dénoté par ces unités lexicales, par exemple : [partie du corps $]+[$ matière $]+[$ nombre de pièces $]+\cdots$

${ }^{(53)}$ https://www . europeana.eu/portal/fr/record/2064108/Museu_ProvidedCHO_ Skulpturensammlung_und_Museum_f_r_Byzantinische_Kunst__Staatliche_Museen_zu_ Berlin_1436661.html. Provenance : Skulpturensammlung und Museum für Byzantinische Kunst, Staatliche Museen zu Berlin, Berlin. Identifier : 3261 (Inventarnummer) | 1436661. Institution : Skulpturensammlung und Museum für Byzantinische Kunst, Staatliche Museen zu Berlin

${ }^{(54)}$ On distingue la définition d'un concept qui n'est pas ambigüe - combinaison unique de caractéristiques -, de la classification d'un objet sous un concept qui peut être «floue », par exemple déterminer si tel vêtement représenté sur telle peinture couvre ou non les genoux. 
définition des axes d'analyse, des caractéristiques essentielles qui les composent, ainsi que leurs dépendances éventuelles (les caractéristiques essentielles ne sont pas nécessairement indépendantes les unes des autres; ainsi, on ne peut s'intéresser au nombre d'attaches que si le vêtement est porté attaché et non pas enroulé autour du corps) s'effectue à l'aide de l'éditeur d'axes d'analyse.

La définition des attributs, caractéristiques descriptives telles que la couleur, le type de fibre et le type de tissage, ainsi que la définition des relations et de leurs propriétés, peuvent se faire à cette étape ou lors d'une phase ultérieure, à l'aide de des éditeurs d'attributs et de relations.

\subsubsection{Tâche 3 : la mise en relation terme - concept}

Éditeur Fast : La troisième étape est au cœur du processus de construction de l'ontoterminologie. Elle consiste à lier termes et concepts sur le principe que nous avons vu précédemment, à savoir qu'un terme désigne un ensemble de caractéristiques essentielles suffisamment «stable » pour être nommé en langue, c'est-à-dire, pour chaque terme précédemment créé, à sélectionner à l'aide d'un éditeur spécifique (éditeur Fast), l'ensemble des caractéristiques essentielles que l'on souhaite associer au terme. Cet éditeur propose à l'expert uniquement les caractéristiques possibles en prenant en compte les relations de compatibilité et de dépendance. S'il n'existe pas de concept correspondant, TEDI propose de créer un nouveau concept dont le nom est construit à partir des caractéristiques essentielles et dont la définition est l'ensemble des caractéristiques sélectionnées par l'expert. En reprenant notre exemple, le terme « exomis » en anglais dénote les caractéristiques essentielles suivantes : /male/, /around body/, /more than one part/, /with sewing/, /without sleeves/, /attached/, /one attachment/, /knee-length/, /unpleated/, /under/. Cet ensemble de caractéristiques constitue la définition formelle du concept dénoté par le terme «exomis » et dont le nom proposé par TEDI est $<$ Garment male around body more than one part with sewing without sleeves attached one attachment knee-length unpleated under $>^{(55)}$.

Il suffit ensuite de préciser, que ce soit à l'aide de l'éditeur de termes de TEDI ou

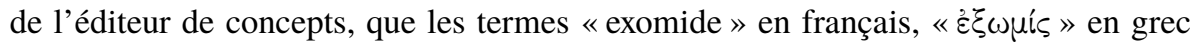
ancien, et « $\varepsilon \xi \omega \mu i \delta \alpha »$ en grec moderne dénotent le même concept.

La gestion des axes d'analyse, de leurs dépendances et de la compatibilité des caractéristiques essentielles est prise en charge par l'environnement TEDI. Elle permet également de guider l'expert dans la sélection des caractéristiques essentielles en ne proposant que celles qui sont à un moment donné possibles.

${ }^{(55)}$ Rappelons que si le terme est donné et est soumis au principe d'économie de la langue, le nom du concept est quant à lui construit de telle sorte qu'en le lisant on comprend la place du concept dans le système notionnel ou du moins la nature des objets qui tombent sous le concept. La «longueur» du nom du concept n'est en rien un handicap dans la mesure où en discours ce sont les termes qui sont utilisés. 


\subsubsection{Tâche 4 : la dimension conceptuelle}

Éditeur de concepts : L'étape suivante concerne la mise à jour de l'ontologie en insérant le concept nouvellement créé dans le système conceptuel. C'est-à-dire par sa mise en relation, hiérarchique ou associative, avec d'autres concepts, et en complétant si besoin sa description par l'ajout de caractéristiques descriptives. Le cas échéant de nouveaux concepts peuvent être introduits à des fins d'organisation du système conceptuel sans qu'il existe pour autant de termes les désignant dans un système linguistique donné(56). Afin d'aider à la structuration du système, TEDI indique automatiquement les concepts génériques possibles pour un concept donné afin de le placer «au bon endroit» dans la hiérarchie conceptuelle.

\subsubsection{Tâche 5 : la dimension linguistique}

Éditeur de termes : Il reste à compléter la dimension linguistique de la terminologie en précisant le statut du terme, sa catégorie grammaticale, ses variations terminologiques, en ajoutant contextes et notes, et en éditant si besoin la définition en langue naturelle générée par TEDI. Cet article ne portant pas sur la dimension linguistique-discursive de notre terminologie, nous ne détaillerons pas davantage cette tâche (voir § 5.2.3 et figure 5.2).

\subsection{Analyse des RÉsultats}

Les résultats obtenus ont été validés par l'expert en comparant les définitions des termes avec les définitions de chose et les représentations figurées sur les sculptures, bas-reliefs, peintures, vases et monnaies, des vêtements généralement associés à ces termes, et accessibles à partir des ressources présentées au chapitre 2.2.

L'objectif de notre démarche n'est pas tant d'imposer des définitions, que de proposer des définitions en langue naturelle qui reposent sur les connaissances du domaine et qui en constituent la justification. Cette approche permet aux experts de débattre de manière objective sur la base des caractéristiques essentielles sur lesquelles en général ils s'accordent. En reprenant notre exemple de l'exomide, nous avons abouti aux définitions suivantes, en anglais, français et grec moderne. « exomis »: Short and non-pleated garment for man, usually worn around the body directly on the skin, this sleeveless garment consists of two pieces of cloth sewn together along the sides, attached on the left shoulder leaving the right shoulder and part of the chest naked. «Exomide»: Vêtement de corps pour homme, court, non-plissé et sans manches. Composé de deux pièces cousues le long des côtés, attaché sur l'épaule gauche laissant l'épaule droite et une partie de la poitrine nues, il est généralement porté directe-

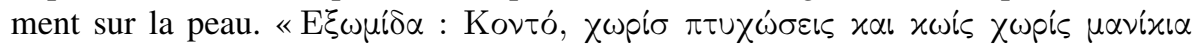

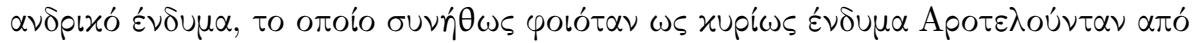

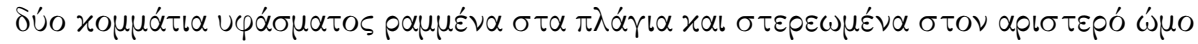

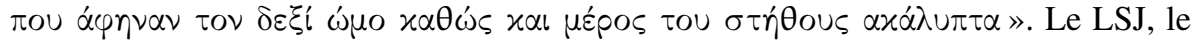

\footnotetext{
${ }^{(56)}$ Rappelons le réseau de termes et le réseau de concepts ne se superposent pas.
} 
dictionnaire bilingue grec-anglais communément utilisé dans le domaine des études classiques, définit l'exomide simplement comme une «tunic with one sleeve«. Cette définition est non seulement incomplète mais problématique en ce qui concerne la notion de «manche $»[58]$.

Enfin, si certaines dénominations sont éclairantes quant à la chose qu'elles dénotent, ainsi un kimmerikon himation est bien une sorte de himation, ce n'est pas le cas du chiton arrhaphos qui n'est pas un chiton (un chiton est par définition cousu, alors que «arrhaphos » signifie non cousu). C'est sur la base de la définition formelle des concepts dénotés par les termes qu'on peut préciser en quoi ils diffèrent. De même, il est important de pouvoir distinguer entre des termes qui seraient des termes de référence, et si nécessaire d'en proposer de nouveaux (néologismes), de ceux d'un usage commun pouvant induire en erreur, ce que permet la notion de statut du terme (préféré, alternatif, etc.).

\subsection{DictionnAire Électronique}

TEDI permet d'exporter les ontoterminologies dans différents formats (voir chapitre 5.2.4). L'exemple ci-dessous est l'export au format e-Dictionary (HTML dynamique) qui permet de visualiser directement l'ontoterminologie dans ses deux dimensions, linguistique et conceptuelle, incluant les informations multimédias associées aux concepts et donc partagées par toutes les terminologies.

\section{Conclusion}

Il ne peut y avoir communication, et à plus forte raison partage et échange de connaissances, que si les experts s'accordent sur les termes à employer et leur définition. Dans le cadre des objets culturels, la signification des termes, en dehors de leur emploi en discours, repose sur les connaissances du domaine et plus précisément sur la façon dont les experts classifient, organisent et structurent les objets du monde. La définition des termes désignant des objets culturels est une définition de chose, c'est-à-dire une définition du concept dont relèvent les objets désignés par le terme. S'accorder sur la conceptualisation du domaine et identifier clairement les termes qui s'y réfèrent sont les deux prérequis à tout travail dans le domaine. Sur ce point, l'ontologie, au sens de l'ingénierie des connaissances, constitue une des voies les plus prometteuses pour les Humanités Numériques (HN) et l'Héritage Culturel (HC). Dans un contexte de partage et d'interopérabilité des données, l'utilisation d'un langage formel et computationnel pour la représentation du système conceptuel garantit une certaine objectivité dans la définition des concepts et permet l'opérationnalisation des terminologies à des fins d'opérationnalisation. Mais l'utilisation d'un langage à la syntaxe et à la sémantique clairement définies ne garantit pas pour autant le consensus sur les connaissances ainsi modélisées, a fortiori si les principes épistémologiques véhiculés par le langage ne correspondent pas à ceux mobilisés par l'expert dans le cadre du processus de modélisation. Experts et ingénieurs de la connaissance ne conceptualisent pas nécessairement de la même façon. 


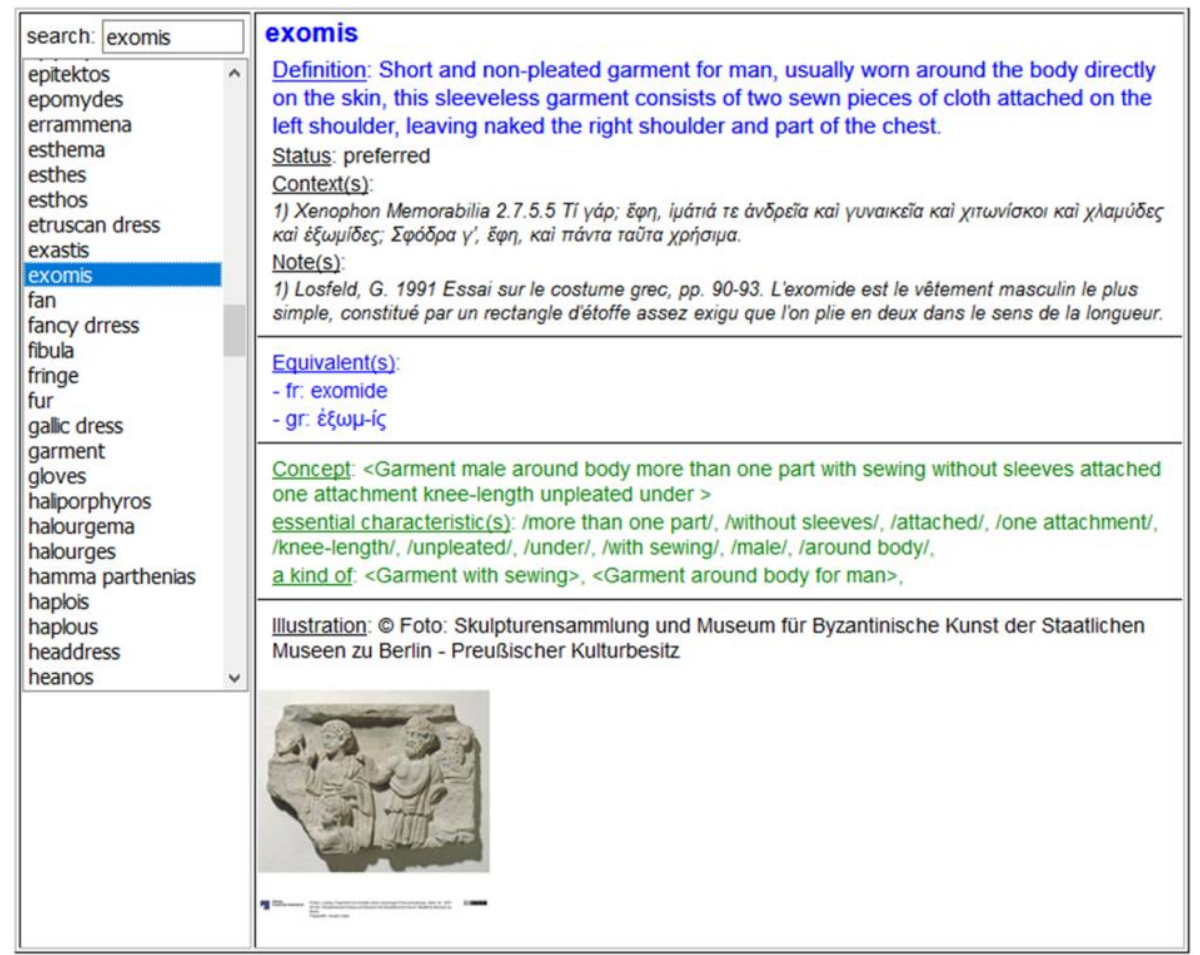

FIgURE 6.2. Export au format e-Dictionary (HTML dynamique)

La construction d'ontologies et de terminologies est une tâche difficile qui nécessite des méthodes outillées spécifiques. La méthode que nous proposons, issue de travaux menés dès 2011 dans le domaine de l'Héritage Culturel, prend en compte la façon de penser et de travailler des experts du domaine. Elle repose sur la notion de caractéristique essentielle sur laquelle se fonde les définitions du concept et du terme en Terminologie, et redonne au terme, en tant que désignation d'un ensemble de caractéristiques suffisamment «stable » pour être nommé en langue, une place importante dans le processus de construction du système conceptuel. Cette méthode est outillée à l'aide de TEDI, éditeur d'ontoterminologies multilingues, qui, grâce à une représentation formelle de l'ontologie, guide l'expert dans sa construction de l'ontoterminologie en ne proposant à chaque étape que les options possibles vérifiant les propriétés logiques du système. La gestion de la dimension linguistique est également prise en compte. La génération d'un pattern de définition en langue naturelle du terme sur la base de la définition formelle du concept dénoté par le terme, tout comme le calcul automatique des équivalents, des hyperonymes et synonymes terminologiques, en sont des exemples. à des fins de partage et d'interopérabilité, les ontoterminologies sont exportées dans des formats d'échange. 
Nous avons choisi comme domaine d'application et de validation celui des vêtements de la Grèce antique, d'abord parce que le vêtement est un sujet d'étude dont l'importance pour l'étude d'une civilisation n'est plus à montrer, mais aussi parce qu'il existe un «flou» terminologique qui nuit grandement aux études classiques et à la communication entre les experts, et qui justifie l'utilisation d'une méthode outillée. Le résultat est un dictionnaire terminologique multilingue structuré selon l'ontologie du domaine et respectant les principes des normes ISO de la Terminologie et les standards du W3C.

Au-delà de simplement appliquer des techniques et méthodes issues de l'Intelligence Artificielle aux Humanités Numériques et à la Terminologie, le travail présenté dans le cadre de cet article illustre tout l'intérêt d'une fertilisation croisée entre ces trois disciplines.

\section{Bibliographie}

[1] G. Aguado-de-Cea, E. Montiel-Ponsoda, M. Poveda-Villalón \& O. X. Giraldo-Pasmin, « Lexicalizing Ontologies : The Issues Behind the Labels », Procedia - Social and Behavioral Sciences 212 (2015), p. 151-158.

[2] X. Aimé, S. George \& J. Hornung, «VETiVOC, une Ressource termino-ontologique modulaire du domaine du Textile, de la Mode et de l'Habillement », Revue d intelligence artificielle 28 (2014), $\mathrm{n}^{\circ} 6$, p. 689-728.

[3] B. Almeida, C. Roche \& C. Rute, «Terminology and ontology development in the domain of Islamic archaeology », in TKE 2016-12th International conference on Terminology and Knowledge Engineering (Copenhagen), 2016, p. 147-156.

[4] R. Arp, B. Smith \& A. D. Spear, Building Ontologies with Basic Formal Ontology, The MIT Press, 2015.

[5] F. Baader, D. Calvanese, D. L. McGuinness, D. Nardi \& P. F. Patel-Schneider, The Description Logic Handbook: Theory, Implementation, and Applications, Cambridge University Press, Cambridge, 2003.

[6] A. BAILly, Dictionnaire grec-français, 26 éd., Paris, 1963, par Séchan L. et Chantraine P., réimpression 2000.

[7] H. Balfet, Y. Broutin \& Y. Delaporte, «Un essai de système descriptif du vêtement », in Vêtement et sociétés 2, L'Ethnographie (Paris), vol. 92-94, Société d'ethnographie de Paris, 1984, p. 363-373.

[8] H. Balfet \& S. Desrosiers, « Où en sont les classifications techniques? », Techniques \& Culture 10 (1988), p. 207-212.

[9] P. BAŃSKI, J. Bowers \& T. ERJAVEC, «TEI-Lex0 guidelines for the encoding of dictionary information on written and spoken forms », in Electronic Lexicography in the 21st Century : Proceedings of ELex 2017 Conference (Leiden, Netherlands), 2017.

[10] R. Barthes, Système de la Mode, Éditions du Seuil, Paris, 1967.

[11] A. Bellandi, E. Giovannetti \& S. Piccini, «LexO : a Lemon-based Collaborative Editor of Lexical, Terminological and Ontological Resources », in The XVIII EURALEX International Congress : Lexicography in Global Contexts Book of Abstracts (Ljubljana, Slovenia), 2018.

[12] A. Bellandi, E. Giovannetti, S. Piccini \& A. Weingart, «Developing LexO : a Collaborative Editor of Multilingual Lexica and Termino-Ontological Resources in the Humanities », in Proceedings of Language, Ontology, Terminology and Knowledge Structures Workshop (LOTKS 2017) (Montpellier), 2017.

[13] D. Bilidas, M. Theologou \& V. Karkaletsis, «Enriching OWL Ontologies with Linguistic and User-Related Annotations : The ELEON System », in Proceedings of the 19th IEEE International Conference on Tools with Artificial Intelligence(ICTAI 2007), 2007, p. 464-467. 
[14] R. J. Brachman \& H. J. Levesque, Readings in Knowledge Representation, Morgan Kaufmann Publishers Inc., San Francisco, CA, USA, 1985.

[15] P. Buitelaar, P. Cimiano \& B. Magnini, Ontology Learning from Text : Methods, Evaluation and Applications, Frontiers in Artificial Intelligence and Applications, vol. 123, Ios Press, Amsterdam, 2005, p. 3-12.

[16] P. Buitelaar, P. Cimiano, J. McCrae, E. Montiel-Ponsoda \& T. Declerck, « Ontology Lexicalisation : The lemon Perspective », in Proceedings of theWorkshops 9th International Conference on Terminology and Artificial Intelligence (Paris), 2011, p. 33-36.

[17] S. Cassagnes-Brouquet \& C. Dousset-Seiden, «Genre, normes et langages du costume », https: //journals.openedition.org/clio/10714, 2012.

[18] J. Cauliez, G. Delaunay \& V. Duplan, « Nomenclature et méthode de description pour l'étude des céramiques de la fin du Néolithique en Provence », Préhistoires méditerranéennes 10-11 (2002), p. 61-81.

[19] P. Chantraine, Dictionnaire étymologique de la language grecque. Histoire des mots, tomes i-ii, Klincksieck, Paris, 1968-1970.

[20] L. Cleland, G. Davies \& L. Llewellyn-Jones, Greek and Roman Dress from A to Z, Routledge, Abington, UK, 2007.

[21] O. Corcho, M. Fernández-López \& A. Gómez-Pérez, « Methodologies, tools and languages for building ontologies. Where is their meeting point? », Data \& Knowledge Engineering $\mathbf{4 6}(2003), \mathrm{n}^{\circ} 1$, p. 41-64.

[22] G. CRAne, «Design Sprint for Perseus 5.0/Open Greek and Latin », 2017, Perseus Digital Library Updates, https://sites.tufts.edu/perseusupdates/2017/07/22/ design-sprint-for-perseus-5-0open-greek-and-latin/.

[23] G. Crane, B. Seales \& M. Terras, «Changing the Center of Gravity : Transforming Classical Studies through Cyberinfrastructure », Digital Humanities Quarterly 3 (2009), no 1, http://www. digitalhumanities.org/dhq/vol/3/1.

[24] C.-V. Daremberg \& E. Saglio, Dictionnaire des Antiquités Grecques et Romaines, Hachette Livre, Paris, 1877-1919.

[25] Y. Delaporte, «Pour une anthropologie du vêtement», in Vêtement et sociétés 1, Actes des Journées de rencontre des 2 et 3 mars 1979 (Paris), Laboratoire d'ethnologie du musée national d'histoire naturelle, Société des amis du Musée de l'Homme, 1981, p. 3-13.

[26] M. Doerr, « Ontologies for Cultural Heritage », in Handbook on Ontologies, Cham, Switzerland, Springer International Publishing, second éd., 2009, p. 463-480.

[27] M. Doerr \& P. LeBoeuf, « Modelling Intellectual Processes : The FRBR — CRM Harmonization », Annual CIDOC Conference, Gothenburg, Sweden, 2006.

[28] M. Dzbor \& E. MotтA, «Engineering and Customizing Ontologies », in Ontology Management. Semantic Web, Semantic Web Services, and Business Applications, Semantic Web and Beyond Computing for Human Experience, vol. 7, Berlin, Springer, 2008, p. 25-57.

[29] E. Eckard, L. Barque, A. NASr \& B. Sagot, « Dictionary-Ontology Cross-Enrichment Using TLFi and WOLF to enrich one another », in CogALex-III - 3rd Workshop on Cognitive Aspects of the Lexicon (Mumbai, India), 2012.

[30] H. Felber, Manuel de terminologie, Infoterm, Unesco, Paris, 1984.

[31] M. Fernández-López, « Overview of Methodologies for Building Ontologies », in IJCAI-99 Workshop on Ontologies and Problem-Solving Methods (Stockholm, Sweden), 1999.

[32] M. Fernández-López, A. Gómez-Pérez \& N. Juristo, «METHONTOLOGY : from Ontological Art towards Ontological Engineering », in Proceedings of the AAAI97 Spring Symposium, 1997, p. 33-40.

[33] M. Genesereth \& R. FiKes, Knowledge Interchange Format, Version 3.0. Reference Manual, Computer Science Department, Stanford University, TR Logic-92-1, 1992.

[34] F. Gherchanoc \& V. Huet, Vêtements antiques : s'habiller, se déshabiller dans les mondes anciens, Errance, Arles, 2012.

[35] P. Gilliver, The making of the Oxford English Dictionary (OED), Oxford University Press, Oxford, 2016.

[36] M. Gras, «Donner du sens à l'objet. Archéologie, technologie culturelle et anthropologie », Annales. Histoire, Sciences Sociales $\mathbf{5 5}^{\mathbf{e}}$ année (2000), n 3, p. 601-614. 
[37] F.-M. Grau, «Introduction », in Histoire du costume, Presses Universitaires de France, Paris, 2007.

[38] A. J. Greimas, La mode en 1830. Langage et société : écrits de jeunesse, Presses Universitaires de France, Paris, 2000.

[39] T. Gruber, «Toward Principles for the Design of Ontologies Used for Knowledge Sharing », International Journal of Human-Computer Studies 43 (1995), n 5-6, p. 907-928.

[40] T. R. GRUBER, «A translation approach to portable ontology specifications », Knowledge Acquisition 5 (1993), n ${ }^{\circ}$ 2, p. 199-220.

[41] M. GrünInger \& M. Fox, «Methodology for the Design and Evaluation of Ontologies », Workshop on Basic Ontological Issues in Knowledge Sharing, Montreal, Canada, 1995.

[42] N. Guarino, D. Oberle \& S. StaAb, «What Is an Ontology? », in Handbook on Ontologies (S. Staab $\&$ R. Studer, éds.), International Handbooks on Information Systems, Springer, Berlin, Heidelberg, 2009, p. 1-17.

[43] G. Hirst, «Ontology and the Lexicon», in Handbook on Ontologies (S. Staab \& R. Studer, éds.), International Handbooks on Information Systems, Springer, Berlin, Heidelberg, 2009, p. 269-292.

[44] M. Horridge, T. Tudorache, J. Vendetti, C. I. Nyulas, M. A. Musen \& N. F. Noy, «Simplified OWL Ontology Editing for the Web : Is WebProtégé Enough?», Lecture Notes in Computer Science, vol. 8218, p. 200-215, Lecture Notes in Computer Science, Springer, Berlin, Heidelberg, 2013, Proceedings part I - 12th International Semantic Web Conference, Sydney New South Wales, Australia.

[45] N. Ide \& J. Veronis, «Extracting knowledge-bases from machine-readable dictionaries : Have we wasted our time? », Proc KB\&KB'93 Workshop, 1993.

[46] ISO 1087-1:2000 Terminology work - Vocabulary - Part 1 : Theory and application.

[47] ISO $704: 2009$ Terminology work - Principles and methods.

[48] ISO 24156-1 2014 « Notations graphiques pour la modélisation des concepts en terminologie et ses relations avec UML - Partie 1 : Lignes directrices pour l'application de la notation UML dans le travail terminologique».

[49] W.-Y. Kıм, « On the Standardization of Ceramic Terminology », Current Anthropology 11 (1970), $\mathrm{n}^{\circ} 2$, p. 168-168.

[50] S. Kirkland Lothrop, Pottery of Costa Rica and Nicaragua, Errance, New York, 2026.

[51] M. M. LeE, «The Peplos and the "Dorian Question" », in Ancient Art and Its Historiography, Cambridge University Press, Cambridge, 2003, p. 118-147.

[52] — - «Problems in Greek Dress Terminology : Kolpos and apoptygma », Zeitschrift für Papyrologie und Epigraphik 150 (2004), p. 221-224.

[53] - Body, Dress, and Identity in Ancient Greece, Cambridge University Press, Cambridge, 2015.

[54] D. B. Lenat \& R. V. Guha, Building Large Knowledge-Based Systems : Representation and Inference in the Cyc Project, Addison-Wesley, Boston, 1990.

[55] H. Liddell, R. Scott \& H. S. Jones (LSJ), A Greek-English Lexicon, Clarendon Press, Oxford, 1996, Ninth edition with a revised supplement.

[56] L. Llewellyn-Jones, Aphrodite's Tortoise : The Veiled Woman of Ancient Greece, Classical Press of Wales, Swansea, 2002.

[57] - Women's Dress in the Ancient Greek World, Classical Press of Wales, Swansea, 2003.

[58] G. Losfeld, Essai sur le costume grec, éditions de Boccard, Paris, 1991, Avec 8 planches de l'auteur, Préface de François Chamoux.

[59] J. P. McCrae, J. Bosque-Gil, J. Gracia, P. Buitelaar \& C. Philipp, «The OntoLex-Lemon Model : Development and Applications », in Electronic Lexicography in the 21st Century : Proceedings of ELex 2017 Conference (Leiden, Netherlands), 2017, p. 587-597.

[60] M. Minsky, «A Framework for Representing Knowledge », 1974, memo 306, Massachusetts Institute of Technology, AI Laboratory.

[61] T. Özacar, Ö. Öztürk \& M. O. ÜNAlir, « ANEMONE : An environment for modular ontology development », Data \& Knowledge Engineering 70 (2011), n ${ }^{\circ}$ 6, p. 504-526.

[62] M. Papadopoulou \& C. Roche, «Le lexique des textiles dans les papyrus de Zénon. Une approche conceptuelle », 2016, Le lexique du mobilier. International Conference IFAO-Cairo.

[63] — «Ontoterminology of Ancient Greek Garments », in TOTh 2017 — Terminology \& Ontology : Theories and application (Chambéry, France), 2017, p. 73-92. 
[64] — - « Ontologization of Terminology. A worked example from the domain of ancient Greek dress », AIDAinformazioni Journal 36 (2018), nº 1-2, p. 89-107.

[65] W. PAPE, Griechisch-Deutsches Handwörterbuch, Vieweg \& Sohn, Braunschweig, 1880.

[66] I. Paresys, G. Bartoleyns, M. Charpy \& M. Meiss-Even, « Vêtement », in Dictionnaire de l'historien, P.U.F., Paris, 2015.

[67] A. Pekridou-Gorecki, Mode im antiken Griechenland, Beck, Munich, 1989.

[68] D. Poole \& A. Mackworth, Artificial Intelligence. Foundations of Computational Agents, Cambridge University Press, Cambridge, 2010.

[69] F. Rastier, «Ontologie(s)», Revue d'Intelligence Artificielle 18 (2004), no 1, p. 15-40.

[70] A. Rey, La terminologie : noms et notions, Collection « Que sais-je ? », P.U.F., Paris, 1979.

[71] G. Richter \& M. Milne, Shapes and names of Athenian vases, Plantin Press, New York, 1935.

[72] D. Rigaux \& C. Roche, «Pour une renovatio du thesaurus PREALP, au cœur des humanités numériques », in TOTh 2016 - Terminology \& Ontology : Theories and application (Chambéry, France), 2018, p. 121-139.

[73] R. B. Roberts \& I. P. Goldstein, « The FRL Manual », Memo 409, Massachusetts Institute of Technology, AI Laboratory, 1977.

[74] L. J. Roccos, «Back-Mantle and Peplos : The Special Costume of Greek Maidens in 4th - Century Funerary and Votive Reliefs », Hesperia 69 (2000), n 2, p. 235-265.

[75] — Ancient Greek Costume : An Annotated Bibliography, 1784-2005, McFarland and Co, Jefferson, N.C, 2006.

[76] C. Roche, «Terminologie et ontologie », Revue Langages 157 (2005), nº 1, p. 48-62.

[77] - «Le terme et le concept : fondements d'une ontoterminologie », in TOTh 2007 — Terminology \& Ontology: Theories and application (Annecy, France), 2007a, p. 1-22.

[78] — - «ire n'est pas concevoir », in Ingénierie des Connaissances (Grenoble, France), CépaduèsEditions, 2007b, p. 157-168.

[79] — - «Should Terminology Principles be re-examined? », in TKE 2012, 10th Terminology and Knowledge Engineering Conference (Madrid, Spain), 2012, p. 17-32.

[80] — , «Ontological definition », in Handbook of Terminology, vol. 1, John Benjamins Publishing, Amsterdam, 2015, p. 128-152.

[81] — - «Une Théorie du Concept pour la Terminologie », à paraître chez les Presses Université Savoie Mont-Blanc, collection Terminologica (Chambéry), 2020.

[82] S. Seppëlä, A. Ruttenberg \& B. Sмith, « Guidelines for writing definitions in ontologies », Ci. Inf. 46 (2017), no 1 , p. 73-88.

[83] G. Simmel, Philosophie der Mode, Pan Verlag, Berlin, 1905.

[84] B. Sismondo Ridgway, «The Fashion of the Elgin Kore », The J. Paul Getty Museum Journal 12 (1984), p. 29-58.

[85] W. Smith, W. Wayte \& G. E. Marindin, A Dictionary of Greek and Roman Antiquities, John Murray, London, 1890.

[86] S. StaAb \& R. Studer, Handbook on Ontologies, second éd., Springer-Verlag, Berlin-Heidelberg, 2009.

[87] K. E. Stears, «Dress and Textiles », in The Edinburgh Companion to Ancient Greece and Rome, Edinburgh University Press, Edinburgh, 2006, p. 226-230.

[88] M. C. Suárez-Figueroa, A. Gómez-Pérez \& M. Fernández-López, « The NeOn Methodology framework : A scenario-based methodology for ontology development », Applied Ontology 10 (2015), p. 107-145.

[89] S. Szulman, «Une nouvelle version de l'outil Terminae de construction de ressources terminoontologiques », IC 2011, $22^{\mathrm{e}}$ Journées francophones d'Ingénierie des Connaissances, Chambéry, 2011.

[90] R. Temmerman, Towards New Ways of Terminology Description. The sociocognitive approach, second éd., John Benjamins, Amsterdam/Philadelphia, 2000.

[91] M. Uschold \& M. Gruninger, «Ontologies : principles, methods and applications », The Knowledge Engineering Review 11 (1996), n², p. 93-136. 
[92] M. Uschold \& M. KING, « Towards a Methodology for Building Ontologies », in In Workshop on Basic Ontological Issues in Knowledge Sharing, held in conjunction with IJCAI-95, AIAI-TR-183, University of Edinburgh, 1995.

[93] B. J. Wielinga, A. T. Schreiber, J. Wielemaker \& J. A. C. Sandberg, «From Thesaurus to Ontology », in Proceedings of the 1st International Conference on Knowledge Capture (New York, NY, USA), Association for Computing Machinery, 2001, p. 194-201.

[94] S. C. Woodhouse, English-Greek Vocabulary. A Vocabulary of the Attic Language, E. P. Dutton, New York, 1910.

[95] J. M. Wright, M. S. Fox \& D. Adam, SRL/1.5 Users Manual. Technical report, Robotics Institute, Carnegie-Mellon University, 1984.

\begin{abstract}
Aвstract. - Classifying objects and choosing the right terms and definitions for them, all the while taking domain knowledge into account, are constant concerns in Digital Humanities (DH). In this regard, as well as in that of data interoperability and sharing on the Web, ontologies, in the sense of Knowledge Engineering (KE), constitute one of the most interesting developments. When conceptualizing a domain, the underlying principles in AI do not necessarily dovetail with those in DH. Adding to that, Terminology, as a discipline, has not really received due attention by AI and DH specialists as yet. This article is interested in the way experts work towards coming up with "definitions of things" and with naming things. The result of this reflection is a tool-assisted method for building terminologies whose conceptual system is a formal ontology, compliant with the ISO and W3C standards. The approach is illustrated by means of presenting the first ontoterminology dictionary of garments of ancient Greece.
\end{abstract}

KEYwords. - Ontology, terminology, ontology building, digital humanities, historical lexicography.

Resumen. - La clasificación de los objetos, la elección de los términos y su definición con respecto al conocimiento del ámbito son preocupaciones constantes en las Humanidades Digitales (HD). En este marco, y en el contexto del intercambio y la interoperabilidad de datos en la Web, las ontologías en el sentido de la Ingeniería del Conocimiento (IC) constituyen uno de los avances más interesantes. Pero los principios que subyacen la conceptualización de un dominio tal como lo entienden la IC y las HD no son necesariamente los mismos. A esto se agrega un problema de Terminología como disciplina que a menudo es subestimada por ambos dominios. Este artículo se interesa a la manera de proceder de los expertos en su "definición de las cosas" y cómo las nombran. Esto nos ha llevado a definir un método basado en herramientas para la construcción de terminologías cuyo sistema conceptual es una ontología formal, en conformidad con las normas de la ISO y los estándares del W3C. Este método está ilustrado gracias al primer diccionario ontoterminológico de la vestimenta de la antigua Grecia

Palabras claves. - Ontología, terminología, construcción de ontología, humanidades digitales, lexicografía histórica.

Manuscrit reçu le 16 septembre 2018, révisé le 22 février 2019, accepté le 16 mars 2019. 